\title{
UV spectra of T Tauri stars from the HST and IUE satellites: BP Tau ${ }^{\star}$
}

\author{
L. Errico ${ }^{1}$, S. A. Lamzin ${ }^{2}$, and A. A. Vittone ${ }^{1}$ \\ 1 Osservatorio Astronomico di Capodimonte, Via Moiariello 16, 80131 Napoli, Italy \\ 2 Sternberg Astronomical Institute, Moscow V-234, 119899, Russia
}

Received 9 April 2001 / Accepted 19 June 2001

\begin{abstract}
Ultraviolet spectra of BP Tau observed with HST/GHRS and IUE satellites were analysed. We found that BP Tau activity can be explained in the frame of a disk accretion model if we assume that the stellar magnetic axis is strongly inclined to the disk plane. The following set of accretion process parameters were derived: relative surface area of the accretion zone $f \simeq 0.25$, accretion rate $\dot{M}_{\mathrm{ac}} \simeq 3.6 \times 10^{-8} M_{\odot} \mathrm{yr}^{-1}$, accretion energy flux $\mathcal{F}=2.3 \times 10^{10} \mathrm{erg} \mathrm{s}^{-1} \mathrm{~cm}^{-2}$ and accretion luminosity $L_{\mathrm{ac}} \simeq 0.37 L_{\odot}$. The relevance of these parameters is discussed. We argue that the Calvet \& Gullbring (1998) accretion shock model is too crude to believe that the accretion spot surface area is indeed proportional to the square of the accretion rate, as Ardila \& Basri (2000) found through this model. A strong flare in the Fe II 2811.8, 2812.1 $\AA$ lines was detected, it was probably produced by an increase of the accretion rate. During the flare, the accretion luminosity was comparable to or even larger than the stellar bolometric luminosity.
\end{abstract}

Key words. stars: pre-main sequence - stars: individual: BP Tau - ultraviolet: stars - X-rays: stars

\section{Introduction}

BP Tau is a classical T Tauri star (CTTS): its H $\alpha$ equivalent width varies from $47 \AA$ (Cabrit et al. 1990) up to $202 \AA$ (Muzerolle et al. 1998). These variations are accompanied by large continuum flux variations: for example the stellar $B$-magnitudes vary from $10.7^{\mathrm{m}}$ to $13.6^{\mathrm{m}}$ (Herbig \& Bell 1988). BP Tau is a single star at least down to $0.01^{\prime \prime}$ (Bernacca et al. 1995). According to Johns-Krull (1999b) the effective temperature of the star is $4060 \mathrm{~K}$ (spectral type $\mathrm{K} 7$ ) and $v \sin i=10.2 \pm 1.8 \mathrm{~km} \mathrm{~s}^{-1}$.

It is usually assumed that the distance to BP Tau is $D=140 \mathrm{pc}$, i.e. equal to the average distance to the Taurus star forming region. With this value and $A_{V} \simeq$ $0.5^{\mathrm{m}}$ Gullbring et al. (1998) found that the luminosity and the radius of the star are $L_{\star} \simeq 0.93 L_{\odot}$ and $R_{\star} \simeq 2.0 R_{\odot}$ respectively. However according to HIPPARCOS measurements the distance to BP Tau is equal to $53_{-11}^{+17} \mathrm{pc}$ (ESA 1997).

Simon et al. (2000) found from IRAM interferometer observations that BP Tau is surrounded by a circumstel-

Send offprint requests to: L. Errico,

e-mail: errico@na.astro.it

* Based on observations with the NASA/ESA Hubble Space Telescope, obtained at the Space Telescope Science Institute, which is operated by the Association of Universities for Research in Astronomy, Inc., under NASA contract NAS 526555 . lar disk with external radius $R_{\mathrm{d}}=108 \pm 4$ AU and inclination $i_{\mathrm{d}}=30_{-2}^{+4}$ degrees to the line of sight. They also found from their data that the mass of BP Tau is $M_{\star}=1.24_{-0.3}^{+0.2} M_{\odot}$ if $D=140 \mathrm{pc}$. Within the approach they used to derive stellar mass, $M_{\star} \propto D$, so the $L_{\star} / M_{*}^{2}$ ratio should not depend on the distance. From a comparison of this value with different theoretical track calculations $M_{\star}$ should be close to $0.8 M_{\odot}$. Thus Simon et al. (2000) concluded that "the distance to the star may be closer than $140 \mathrm{pc}$ but not as extreme as the HIPPARCOS value".

In this study we accept the following set of BP Tau parameters: $M_{\star}=0.8 M_{\odot}, R_{\star}=2 R_{\odot}$ and $A_{V}=0.5^{\mathrm{m}}$. The stellar radius is the only value in this set which depends on the distance: $L_{\star} \propto D^{2}$ so $R_{\star} \propto D$ for a fixed $T_{\text {eff }}$. The main quantitative results of our paper are not sensitive to the current uncertainty in the value of $D$. For example the escape velocity $V_{\infty}=(2 G M / R)^{1 / 2} \simeq$ $390(140 / D)^{1 / 2} \mathrm{~km} \mathrm{~s}^{-1}$ and the relative surface area of the accretion zone derived from Eq. (1) do not depend on the distance at all.

A clear signature of circumstellar matter accretion was found in the optical spectrum of BP Tau - see Mundt \& Giampapa (1982), Edwards et al. (1994), Fernandez et al. (1995), Johns \& Basri (1995), Muzerolle et al. (1998), Gullbring et al. (1996, 1998), Alencar \& Basri (2000). Johns-Krull et al. (1999b) measured the strength of the 
Table 1. Log of HST Program 3845 on 30 July 1993.

\begin{tabular}{llllll}
\hline$t_{0}$, UT & Dataset & Target & Grating & $\Delta \lambda, \AA \AA$ & $N$ \\
\hline $17: 33$ & z18e0103t & W_Cal & G270M & $2878-2924$ & 1 \\
$17: 34$ & z18e0104t & BP Tau & G270M & $2777-2823$ & 2 \\
$17: 46$ & z18e0105t & W_Cal & G160M & $1503-1539$ & 1 \\
$17: 47$ & z18e0106t & W_Cal & G160M & $1384-1402$ & 1 \\
$17: 49$ & z18e0107t & BP Tau & G160M & $1383-1419$ & 6 \\
$19: 08$ & z18e0108t & BP Tau & G160M & $1532-1568$ & 5 \\
\hline
\end{tabular}

average magnetic field finding $B=2.1 \pm 0.3 \mathrm{kG}$. Furthermore Johns-Krull et al. (1999a) found strong circular polarization in the He I 5876 emission line, indicating a mean longitudinal magnetic field of $\simeq 2.5 \mathrm{kG}$ in the line formation region. They concluded that "accretion occurs preferentially along large-scale magnetic loops that occupy a small fraction of the stellar surface".

Ardila \& Basri (2000) (AB00) analyzed long wavelength low resolution IUE spectra and found, in the frame of the accretion shock model of Calvet \& Gullbring (1998) (CG98), an average BP Tau accretion rate $\dot{M}_{\mathrm{ac}}=1.6 \times$ $10^{-8} M_{\odot} \mathrm{yr}^{-1}$ such that the accretion zone (spot) occupies $0.4 \%$ of the stellar surface. They also found that the spot area increases as the square of $\dot{M}_{\text {ac }}$ and claimed that "current models of the accretion process fail to reproduce such an effect".

The evidence for a BP Tau stellar wind is relatively poor in the optical band: no blueshifted absorption features have been observed in the high resolution profiles of strong permitted optical lines (e.g. Balmer, Na I D, Ca II, O I or He I). At the same time Gullbring et al. (1996) noted "the blue asymmetric appearances in the profiles of the higher Balmer lines". Usually the centroids of [O I] 6300, [O I] 5577 and [S II] 6731 lines are blueshifted in CTTS spectra indicating a matter outflow with terminal velocity up to $300 \mathrm{~km} \mathrm{~s}^{-1}$. But in the case of BP Tau the centroids of [O I] lines are redshifted and the [S II] 6731 line profile is entirely situated between +100 and $+200 \mathrm{~km} \mathrm{~s}^{-1}$ (Hartigan et al. 1995).

In the frame of our detailed analysis of CTTS UV spectra (Errico et al. 2000; Lamzin 2000a, 2000b; Lamzin et al. 2001), in this paper we interpret the spectra of BP Tau obtained with HST and IUE satellites in order to derive the main parameters of the accretion shock and outflow/inflow geometry of this star.

\section{Observations}

HST observed BP Tau on 30 July 1993 (Program 3845) in 3 spectral bands with the Goddard High Resolution Spectrograph in the medium resolution mode and Detector 2. The wavelength calibration lamp was used in nearly the same spectral bands. The starting times of each observation, Archive Dataset names, grating designation, observed spectral bands and number of independent exposures (RPTOBS +1 parameter) are presented in Table 1. The spectra were adopted from the HST Archive,

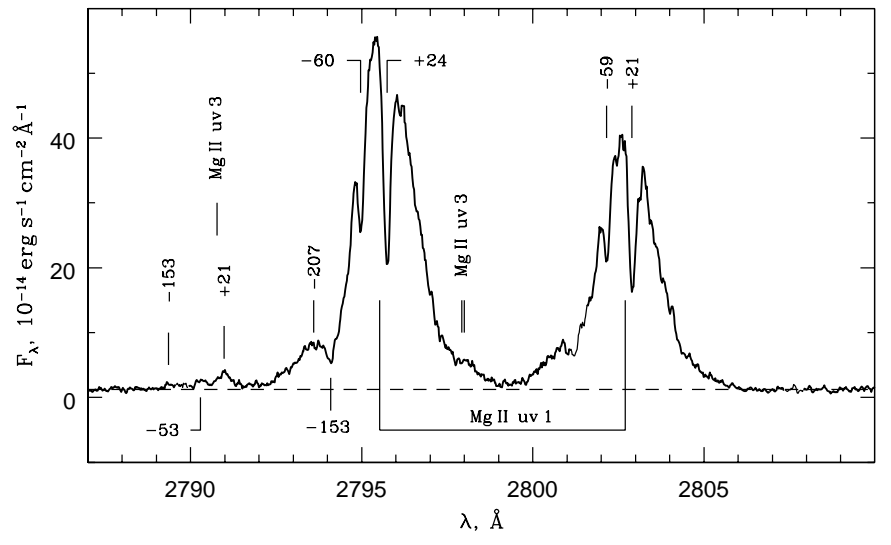

Fig. 1. BP Tau spectrum in the vicinity of Mg II $2800 \AA$ doublet. The radial velocity of main features is shown in $\mathrm{kms}^{-1}$ relative to the rest wavelength.

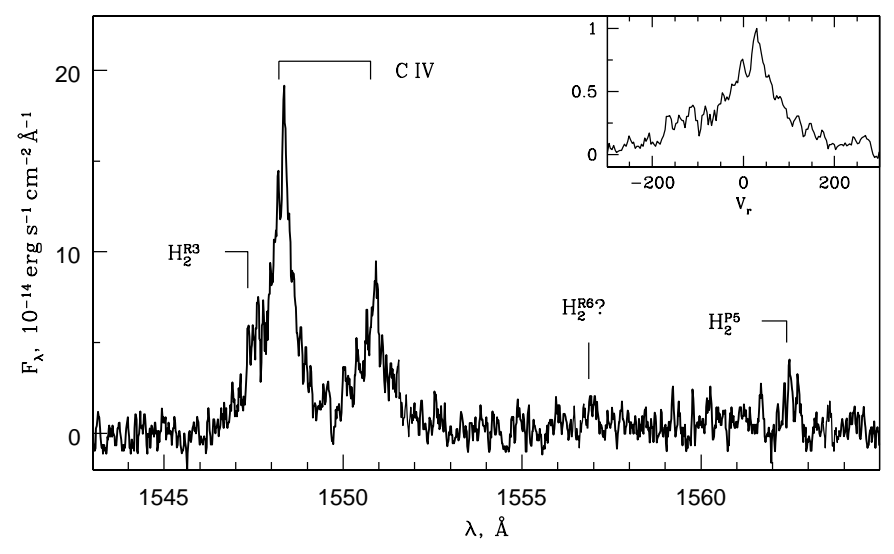

Fig. 2. BP Tau spectrum in the vicinity of C IV $1550 \AA$ doublet. The line profile of the C IV $1548.20 \AA$ is shown in the insert at top right corner. $X$-axis of the insert is labelled in $\mathrm{km} \mathrm{s}^{-1}$.

recalibrated using the most up-to-date reference files and processed with IRAF v2.11 and STSDAS/TABLES v2.0.2 software as recommended in Chapter 36 of the "HST Data Handbook".

The standard "pipeline" wavelength calibration was improved by using the STSDAS waveoff task and respective W_CAL observations - see Table 1 . All wavelengths are corrected for the orbital motion of the HST and the Earth and are presented for vacuum if $\lambda<2000 \AA$ and for air otherwise. The Van Hoof (1999) electronic database was used to identify lines in BP Tau spectra along with an atlas of $\mathrm{H}_{2}$ lines (Roncin \& Launay 1994). To improve the signal-to-noise $(\mathrm{S} / \mathrm{N})$ ratio we combined all independent exposures for each spectral band and additionally smoothed them via a 4-point running mean, so the resulting spectral resolution is near $15 \mathrm{~km} \mathrm{~s}^{-1}$. The five spectral intervals shown by thin lines in Figs. 1-3 indicate the "dead" diodes of Detector 2.

High resolution spectra in the 1850-3350 $\AA$ spectral band observed with IUE satellite were adopted from the INES database (http://ines.oat.ts.astro.it). The spectra were not additionally processed. Archive dataset names, date and universal time of the beginning of each 


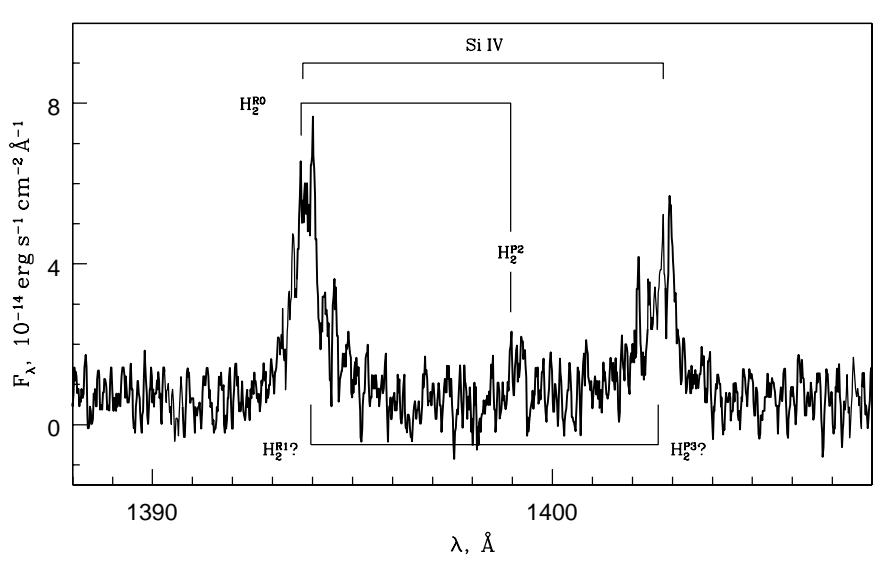

Fig. 3. BP Tau spectrum in the vicinity of Si IV $1400 \AA$ doublet.

observation and exposure time $\Delta t$ (in hours) are presented in Table 2. The last column shows the counts of the IUE fine error sensor (FES), which give an estimation of the stellar visual brightness.

\section{Results}

The spectrum of BP Tau in the 2787-2810 $\AA$ band is shown in Fig. 1. The continuum flux was derived from a featureless part of the spectrum between $2807 \AA$ and $2822 \AA: F_{\mathrm{c}}=1.3 \pm 0.3 \times 10^{-14} \mathrm{erg} \mathrm{s}^{-1} \mathrm{~cm}^{-2} \AA^{-1}$, i.e. $F_{\mathrm{c}}>4 \sigma_{\mathrm{c}}$. This value coincides within $1 \sigma_{\mathrm{c}}$ with the continuum flux derived between $2778 \AA$ and $2788 \AA$. We can see strong $\mathrm{Mg}$ II $\mathrm{h}$ and $\mathrm{k}$ lines with a narrow interstellar (IS) absorption feature slightly redshifted relative to the rest wavelength, in agreement with the BP Tau radial velocity $V_{\mathrm{r}}=15.8 \pm 1.0 \mathrm{~km} \mathrm{~s}^{-1}$ (Hartmann et al. 1986). Two additional absorption features are present in the blue wings of both lines at $\simeq-60$ and $\simeq-150 \mathrm{~km} \mathrm{~s}^{-1}$. The total flux of both Mg II uv1 lines is about $1.8 \times 10^{-12} \mathrm{erg} \mathrm{s}^{-1} \mathrm{~cm}^{-2}$.

The upper levels $3 \mathrm{p}^{2} \mathrm{P}_{1 / 2,3 / 2}^{0}$ of $\mathrm{Mg} \mathrm{II} \mathrm{h}$ and $\mathrm{k}$ resonant lines are also the low levels of the Mg II $u v 3$ triplet. An emission feature is present near the expected position of two lines of the triplet (2797.99 and 2798.06 $\AA$ ), but it is superimposed on the red wing of $\mathrm{Mg}$ II $\mathrm{h}$ line, so it is difficult to say anything about these line profiles. The profile of the third $(\lambda 2790.84 \AA)$ line of the $u v 3 \mathrm{mul}-$ tiplet apparently consists of three emission components. The maximum of the first (more or less symmetric) component is redshifted by $+21 \mathrm{kms}^{-1}$ relative to the rest wavelength. The second component shows a flat top with the center blueshifted by $-53 \mathrm{~km} \mathrm{~s}^{-1}$, i.e. it corresponds to the position of the first absorption feature in the blue wings of $\mathrm{h}$ and $\mathrm{k}$ lines. The third emission component of the $2790.84 \AA$ line seems to be blueshifted by the same value $\left(\simeq-150 \mathrm{kms}^{-1}\right)$ as the "high-velocity" absorption features of the resonant lines. However a few pixels between the second and the third components of the line fall on to the "dead" diodes of Detector 2, so the red wing of the third component could be a bit distorted - see Fig. 1.
Table 2. High resolution IUE spectra of BP Tau.

\begin{tabular}{lllll}
\hline Dataset & Date & $t_{0}, \mathrm{UT}$ & $\Delta t, \mathrm{~h}$ & $\mathrm{FESc}$ \\
\hline LWR 11130 & $1981-07-24$ & $06: 41$ & 3.5 & 218 \\
LWP 06963 & $1985-10-22$ & $02: 00$ & 3.0 & 236 \\
LWP 09282 & $1986-10-10$ & $00: 51$ & 4.5 & 176 \\
LWP 09417 & $1986-10-26$ & $13: 20$ & 4.5 & 162 \\
\hline
\end{tabular}

We did not find any more emission or absorption features in the spectrum with intensity $>2 \sigma_{\mathrm{c}}$, while relatively strong (emission or absorption) lines of the Fe II $u v 234$ multiplet are present in analogous HST/GHRS spectra of RU Lup (Lamzin 2000a), RY Tau (Lamzin 2000b), RW Aur (Errico et al. 2000) and DF Tau (Lamzin et al. 2001).

The spectrum of BP Tau in the vicinity of the C IV $1550 \AA$ doublet is shown in Fig. 2. Its $\mathrm{S} / \mathrm{N}$ ratio is rather low, so the continuum is underexposed and we can only identify the lines of $\mathrm{C}^{+3}$ and molecular hydrogen. The flux ratio of the C IV $1550 \AA$ doublet component is close to $2: 1$, and the total flux of the doublet is $\simeq 2.4 \times 10^{-13} \mathrm{erg} \mathrm{s}^{-1} \mathrm{~cm}^{-2}$, this is $4 \%$ less than the average value found by Valenti et al. (2000) from all IUE spectra. The normalized profile of the C IV 1548.20 A line is shown in the insert in the upper right corner of the figure. The line center is redshifted, but it is difficult to judge the redshift value or the reality of the features in the blue wing of the C IV $1550.77 \AA$ line.

$\mathrm{H}_{2}$ lines $\mathrm{R}(3)$ 1547.34 $\AA$ and $\mathrm{P}(5) 1562.39 \AA$ have a common upper level $v^{\prime}=1, J^{\prime}=4$ of the first excited electronic configuration $2 \mathrm{p} \sigma \mathrm{B}^{1} \Sigma_{\mathrm{u}}^{+}$of the molecule. If the lines are optically thin their expected flux ratio is $\simeq 1.1$ (Abgrall et al. 1993), so the R(3) line contributes no more than $10 \%$ to C IV 1550 doublet flux. We identified (but with some doubts) the emission feature at $\lambda \simeq 1557 \AA$ with the $\mathrm{R}(6) 1556.87 \AA$ line (B1-8 transition) of $\mathrm{H}_{2}$.

Some useful information can be extracted from the fact that the $\mathrm{R}(6) 1556.87 \AA$ line is at least twice as weak as the $\mathrm{P}(5) 1562.39 \AA$ line. Pumping of these lines occurs from these levels with a practically identical excitation energy and the absorption coefficients of the pumping transitions are almost the same. Both lines are pumped by quanta from the red wing of $\mathrm{L} \alpha$ line, but the redshifts for the $\mathrm{P}(5)$ and $\mathrm{R}(6)$ lines are $+100 \mathrm{~km} \mathrm{~s}^{-1}$ and $+17 \mathrm{~km} \mathrm{~s}^{-1}$ respectively. We conclude therefore that the $\mathrm{L} \alpha$ line has a deep central depression due to self-absorption as in the case of the solar chromosphere (Mihalas 1978).

Unfortunately the BP Tau spectrum in the vicinity of the Si IV 1400 doublet is also very noisy - see Fig. 3. Except Si IV lines, only the $\mathrm{H}_{2}$ B0-5 P(2) $1398.95 \AA$ line can be identified unambiguously. The $\mathrm{R}(0) 1393.72 \AA$ molecular hydrogen line also has the same upper level and falls into the spectral bands of Figs. 2 and 3. If both lines are optically thin then the $\mathrm{R}(0)$ line flux should be $\simeq 1.9$ times larger than the $\mathrm{P}(2)$ line flux, so it contributes significantly to the observed flux of the SiIV $1393.76 \AA$ line. 


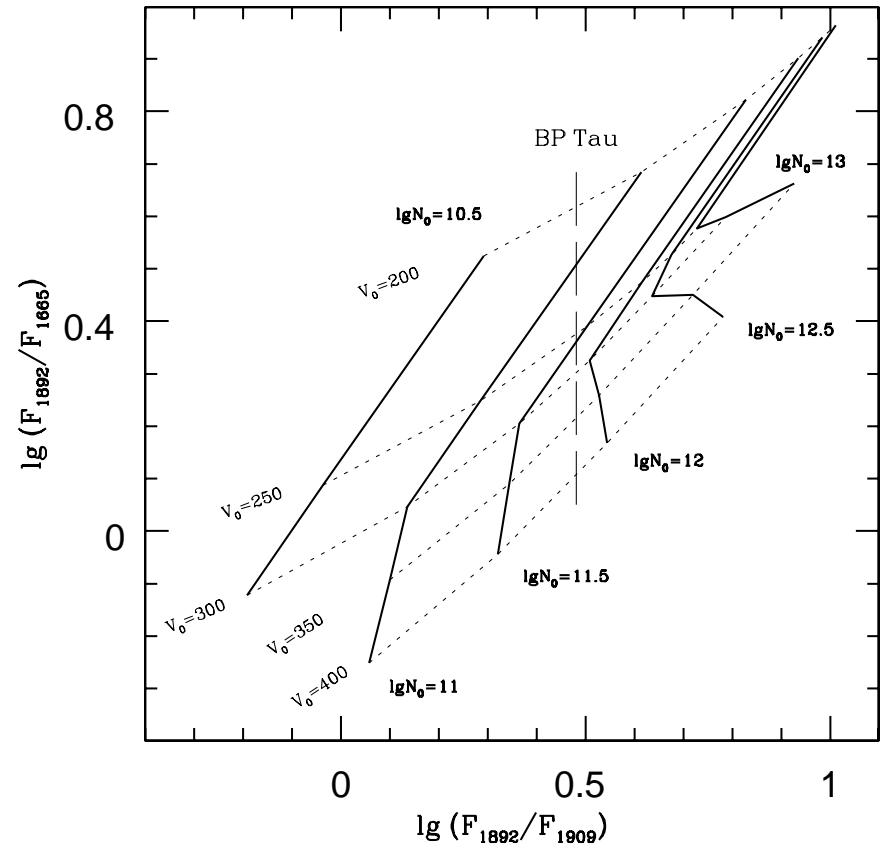

Fig. 4. Semiforbidden flux ratio diagram for CTTS accretion shock. Flux ratios of Si III] 1892, O III] 1665 and C III] 1909 lines are plotted along $X$ and $Y$ axis. See text for details.

One can expect from an analogy with the spectra of RU Lup (Lamzin 2000a), RW Aur (Errico et al. 2000) and DF Tau (Lamzin et al. 2001) that two additional $\mathrm{H}_{2}$ lines will also be superimposed on both lines of Si IV doublet. They are $\mathrm{R}(1) 1393.96 \AA$ and $\mathrm{P}(3) 1402.65 \AA$ lines with $\mathrm{B}(0,2)$ common upper level and a transition probabilities ratio $\simeq 1.4$ (Abgrall et al. 1993). There are some marginal indications that the lines are indeed present in the BP Tau spectrum. If so the real flux of the Si IV doublet can be almost twice as low as the observed value of $8.7 \times 10^{-14} \mathrm{erg} \mathrm{s}^{-1} \mathrm{~cm}^{-2}$. Beside that a number of bad pixels are at the position of Si IV 1393.76 and $1402.77 \AA$ lines, so we will not discuss these.

\section{Discussion}

\subsection{Accretion flow parameters}

The O III] 1665, Si III] 1892 and C III] 1909 semi-forbidden lines originated in the CTTS accretion shock are optically thin and are expected to have comparable intensities (Lamzin 1998). The flux ratio of these lines depends on the infall gas velocity $V_{0}$ and the infall gas particle density $N_{0}$, but does not depend on the geometry of the accretion zone. Thus one can derive $V_{0}$ and $N_{0}$ values (Gomez de Castro \& Lamzin 1999). According to JohnsKrull et al. (2000) the average flux ratio of the C III] 1909 and Si III] 1892 lines derived from low resolution IUE spectra of BP Tau is $0.33 \pm 0.04$. Unfortunately the flux ratio of the O III] 1665 and Si III] 1892 lines is unknown, so in Fig. 4 we plot only the corresponding vertical line. We can see even from this restricted dataset that the average particle density of the infalling gas in BP Tau does not exceed $10^{12} \mathrm{~cm}^{-3}$.

The escape velocity on BP Tau is $V_{\infty} \simeq 390 \mathrm{~km} \mathrm{~s}^{-1}$. Probably the angle between the rotational axis and the circumstellar disk is small or even zero, so the equatorial velocity of the star $V_{\text {rot }} \simeq 20 \mathrm{~km} \mathrm{~s}^{-1}$. Thus the corotational radius $R_{\mathrm{c}}=R_{\star}\left(V_{\infty} / V_{\mathrm{rot}}\right)^{2 / 3} \simeq 7 R_{\star}$. Bearing in mind the large magnetic field strength of the star we can suppose that the accretion radius is close enough to this value so the expected average infall gas velocity $V_{0}$ is not far from $350 \mathrm{~km} \mathrm{~s}^{-1}$. It then follows from Fig. 4 that $N_{0} \simeq 5 \times 10^{11} \mathrm{~cm}^{-3}$. We adopt this value and $V_{0}=350 \mathrm{~km} \mathrm{~s}^{-1}$ as the average parameters of the accretion shock.

The SiIII $1892 \AA$ line originates within the H II preshock zone of which the extension is much less than the stellar radius for a given set of $V_{0}$ and $N_{0}$ parameters (Lamzin 1998). The line formation region can be considered as a plane-parallel slab and the emissivity of the line can be characterized by the specific intensity $I_{\mathrm{n}}$ $\left(\mathrm{erg} \mathrm{s}^{-1} \mathrm{~cm}^{-2}\right.$ ster $\left.^{-1}\right)$ in the direction perpendicular to the shock front. The observed Si III] $1892 \AA$ line flux $F$ can be expressed through this value and the total accretion shock surface area $S_{\mathrm{ac}}$ as follows (Gomez de Castro \& Lamzin 1999):

$F=\frac{I_{\mathrm{n}} S_{\mathrm{ac}}}{2 D^{2}} \xi_{\lambda} \simeq 2.0 \times 10^{-19} f I_{\mathrm{n}}\left(\frac{R_{\star}}{2 R_{\odot}}\right)^{2}\left(\frac{140}{D, \mathrm{pc}}\right)^{2}$

where $\xi_{\lambda}=\exp \left(-0.92 \mathrm{~A}_{\lambda}\right)$ is the interstellar extinction coefficient at $\lambda=1892 \AA$ (it is $\simeq 0.3$ if we assume $A_{V}=$ $0.5^{\mathrm{m}}$ and the standard extinction law, Seaton 1979); $f$ is the relative fraction of the BP Tau surface taken up by the accretion zone. We assume that the total surface area of the accretion zone is two times larger than the observed area - a factor 2 in the denominator of Eq. (1).

The average observed Si III] 1892 line flux is $F \simeq$ $4.1 \pm 0.2 \mathrm{erg} \mathrm{s}^{-1} \mathrm{~cm}^{-2}$ (Valenti et al. 2000) and $I_{\mathrm{n}} \simeq$ $8 \times 10^{5} \mathrm{erg} \mathrm{s}^{-1} \mathrm{~cm}^{-2}$ ster $^{-1}$ for our set of $V_{0}$ and $N_{0}$ parameters - see Fig. 1 of Gomez de Castro \& Lamzin (1999). From Eq. (1) follows $f \simeq 0.25$, i.e. the accretion spot takes up $\sim 25 \%$ of the stellar surface on average. Now we can find the accretion rate, the energy flux $\mathcal{F}$ of the accretion flow and the accretion luminosity from the relations:

$\dot{M}_{\mathrm{ac}}=\rho_{0} V_{0} S_{\mathrm{ac}}, \quad \mathcal{F}=\rho_{0} \frac{V_{0}^{3}}{2}, \quad L_{\mathrm{ac}}=\dot{M}_{\mathrm{ac}} \frac{V_{0}^{2}}{2}$

where $\rho_{0}=\mu_{\mathrm{i}} m_{\mathrm{H}} N_{0}$ is the infall gas density, $m_{\mathrm{H}}$ is the hydrogen atom mass and $\mu_{\mathrm{i}}=1.3$ is the average molecular weight of the infalling gas which we assume to have a cosmic abundance (Allen 1973). It follows that $\dot{M}_{\mathrm{ac}} \simeq 3.6 \times 10^{-8} M_{\odot} \mathrm{yr}^{-1}, \mathcal{F}=2.3 \times 10^{10} \mathrm{erg} \mathrm{s}^{-1} \mathrm{~cm}^{-2}$ and $L_{\mathrm{ac}} \simeq 0.37 L_{\odot}$ i.e. $39 \%$ of the BP Tau bolometric luminosity.

According to CG98 $\dot{M}_{\mathrm{ac}}=2.9 \times 10^{-8} M_{\odot} \mathrm{yr}^{-1}$ and $L_{\mathrm{ac}} \simeq 0.18 L_{\odot}$. The average values of the accretion rate 
and luminosity derived from 45 IUE spectra by AB00 are: $\dot{M}_{\mathrm{ac}}=1.6 \times 10^{-8} M_{\odot} \mathrm{yr}^{-1}, L_{\mathrm{ac}} \simeq 0.09 L_{\odot}$. Our values are about two times larger. This discrepancy can be due to variability and difference in the infall gas velocity adopted by CG98 and AB00, who assumed $M_{\star}=0.49 M_{\odot}$ so they adopted $V_{0}=300 \mathrm{~km} \mathrm{~s}^{-1}$ instead of our $V_{0}=350 \mathrm{~km} \mathrm{~s}^{-1}$. Thus, our CG98 and AB00 values of $L_{\mathrm{ac}}$ and $\dot{M}_{\mathrm{ac}}$ are in a reasonable agreement.

The accretion energy flux and filling factor of the accretion spot found in the quoted papers are also similar to each other: $f=7 \times 10^{-3}, \log \mathcal{F}=11.5$ (CG98) and $\bar{f}=4.4 \times 10^{-3}, \log \overline{\mathcal{F}}=11.7$ (AB00). The coincidence of the results is not surprising because AB00 used the accretion shock model calculated by CD98. Our $f$ and $\mathcal{F}$ values, derived within the frame of the Lamzin (1998) accretion shock model, differ from the AB00 and CG98 values by more than one order of magnitude. Obviously this discrepancy is the result of the different approach used.

As the R/D-ratio does not depend on distance, our accretion shock parameters are also independent on the current distance uncertainty, but they depend on the interstellar extinction value through the coefficient $\xi_{\lambda}$ in Eq. (1). For example if $A_{V}=0.3^{\mathrm{m}}$ instead of $0.5^{\mathrm{m}}$ then our $f, \dot{M}_{\mathrm{ac}}$ and $L_{\mathrm{ac}}$ values would be $\simeq 1.6$ times less. We suppose however that the main shortcoming of our approach is the assumption on the homogeneous character of the accretion. Equation (1) assumes that $I_{\mathrm{n}}$ is the same in all points of the accretion zone, otherwise it should be interpreted as an average value. The same is true for the diagnostic diagram in Fig. 4 used to derive the infall gas density. But if the stellar magnetic axis is strongly inclined to the disk plane (see below) one can expect that $V_{0}$ and $N_{0}$ values cannot be the same at all points of the accretion zone. Therefore we could overestimate the values for f, $\dot{M}_{\mathrm{ac}}$ and $L_{\mathrm{ac}}$, but not by more than 2-3 times.

CG98 derive the accretion shock parameters by comparing the calculated continuum spectral energy distribution with the observed one. The excess of the Balmer continuum emission originates in the upper layers of the heated stellar atmosphere at the bottom of an accretion column. To calculate the spectrum of this region CG98 use a relatively simple approach which gives them the possibility to find only the emergent flux $E_{\nu}$ i.e the specific intensity $I_{\nu}(\theta)$ averaged over cosine of the angle $\theta$ between the line of sight and the normal to the plane-parallel gas slab. Then they fit the observed spectrum varying two parameters: $N_{0}$ and $V_{0}$ or their equivalent. $S_{\text {ac }}$ or $f$ can be derived from the observed absolute flux with a known $\mathcal{F}$ value.

GC98 and AB00 derive the accretion shock parameters "averaged over $\cos \theta "$ - see e.g. AB00 and their Table 3. Meanwhile the BP Tau veiling continuum originates in the region where the temperature increases outwards, so the larger $\theta$ the larger the continuum specific intensity should be. This is also why we observe limb brightening effect in the solar chromosphere (Mihalas 1978). Obviously the average over $\cos \theta$ results in the preferential contribution of specific intensities with low $\theta$ into the resulting $E_{\nu}$-value.
These are the smallest intensities, so if the accretion zone is observed within a large range of $\theta$ then the comparison of an observed spectrum with $E_{\nu}$ results in an overestimation of $\mathcal{F}$ and a corresponding underestimation of $f$ one can derive the same accretion luminosity with a large energy flux density and a small $f$ or vice versa.

The extent of the CIV $1548 \AA$ line wings does not exceed $200 \mathrm{~km} \mathrm{~s}^{-1}$ (Fig. 2) while the infall gas velocity is at least 1.5 times larger. It means that the "average" angle between the line of sight and the accretion zone is large enough, so CG98 and AB00 have overestimated $\mathcal{F}$ and underestimated $f$. What is more, CG98 also assume that the accretion is homogeneous, as we do. It cannot be said without special calculations and a specification of the accretion zone geometry how large the resulting error is. But the CG98 approach seems too crude to believe that the $f \propto \dot{M}_{\mathrm{ac}}^{2}$ dependence found by AB00, applying this model, is real. CG98 found that highly veiled CTTSs have similar energy fluxes $\mathcal{F}$ as less veiled stars but a larger surface coverage $f$.

On the other hand as far as it becomes possible to fit the observed continuum spectral energy distribution more or less well, the value of $L_{\mathrm{ac}}$ appears to be automatically correct. The spread of CTTS infall gas velocity is relatively small $\left(250<V_{0}<400 \mathrm{~km} \mathrm{~s}^{-1}\right)$, so according to Eq. (2) the accretion rate derived by CG98 is likely. In fact our values of $L_{\mathrm{ac}}$ and $\dot{M}_{\mathrm{ac}}$ are in reasonable agreement with CG98's values taking into account the BP Tau variability and the uncertainty in the $V_{0}$ value.

Both our methods and those of GC98 to derive accretion shock parameters of BP Tau are not perfect because they are based on the assumption that accretion is homogeneous. But an additional averaging of the specific intensity over $\theta$ makes it impossible, in principle, to derive more accurate accretion shock parameters within the frame of the GC98 model. In contrast our approach gives the possibility to derive $N_{0}$ and $V_{0}$ values in each point of the accretion zone as well as its geometry if we succeed in observing the star at different phases of the rotational period (Lamzin 2000c).

\subsection{The outflow}

Two absorption features are present in the blue wings of the Mg II $\mathrm{h}$ and $\mathrm{k}$ resonant lines in the HST spectrum. There are also two emission components in the profile of the Mg II $2790.84 \AA$ line blueshifted to the same values: $\Delta V \simeq-60$ and $\simeq-150 \mathrm{~km} \mathrm{~s}^{-1}$. Thus at the moment of observations there were two separate regions of gas moving outwards between the star and the Earth with significantly different radial velocities. We call them low velocity (LV) and high velocity (HV) region.

There are no FeII absorption lines in the spectrum shown in Fig. 1 including uv234 multiplet lines which are expected to be the strongest (Errico et al. 2000). The excitation energy of the $u v 234$ multiplet low level $\left(E_{i} \simeq 3.2 \mathrm{eV}\right)$ is less than the energy of the $\mathrm{MgII}$ $u v 3$ subordinate multiplet emission lines: $h \nu \simeq 4.4 \mathrm{eV}$. 


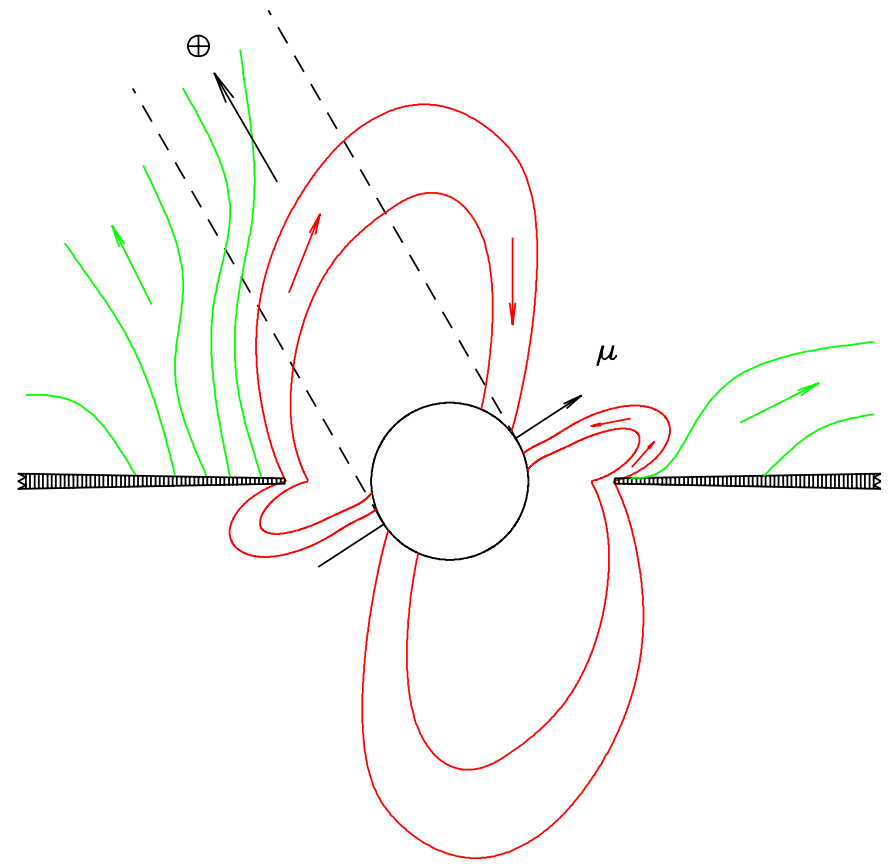

Fig. 5. Qualitative scheme of the geometry of BP Tau inflow and outflow with both open and close magnetic field lines (solid lines). The broken lines show the line of sight.

The absence of Fe II lines in absorption is possible if the gas temperature in both $\mathrm{LV}$ and $\mathrm{HV}$ regions is relatively low and the excitation of the $\mathrm{Mg}$ II $2790.84 \AA$ line is due to radiation rather than to electron collisions. Cold external gas absorbs Mg II $\mathrm{h}$ and $\mathrm{k}$ line quanta produced by an accretion shock resulting in the pumping of ${ }^{2} \mathrm{P}_{1 / 2,3 / 2}^{0}$ levels. Quanta from the red wing of $\mathrm{Mg}$ II $\mathrm{h}$ line excite the ${ }^{2} \mathrm{D}_{3 / 2}$ level and its de-excitation occurs mainly via emission of the $\mathrm{Mg}$ II 2790.84 line $\left({ }^{2} \mathrm{D}_{3 / 2} \rightarrow{ }^{2} \mathrm{P}_{1 / 2}^{0}\right.$ transition $)$.

The Fe I $2788.17 \AA$ line of the $\mathrm{a}^{5} \mathrm{~F}-\mathrm{y}^{5} \mathrm{G}^{0}$ multiplet is also absent in the BP Tau spectrum, while the excitation energy of the $\mathrm{a}^{5} \mathrm{~F}$ term is only $0.86 \mathrm{eV}$ and the $\log g f$ value of the line is $\simeq-0.05$ (Nave et al. 1994), i.e. the line is expected to be strong unless the relative number of iron atoms is small enough. We conclude that the gas in the $\mathrm{LV}$ and $\mathrm{HV}$ regions is cold but that the iron and magnesium are singly ionized. We suppose that the source of ionization is H I L $\alpha$ quanta produced by the accretion shock as in the case of RW Aur (Errico et al. 2000). Indeed the BP Tau $\mathrm{H} \alpha$ line equivalent width is $\sim 100 \AA$, which means that $\sim 1 \%$ of stellar luminosity is radiated in this line and the $\mathrm{L} \alpha$ line is expected to be even stronger.

In this case both LV and HV regions should be close to the star. At the same time the circumstellar disk is seen nearly pole-on: $i_{\mathrm{d}} \simeq 30^{\circ}$ (Simon et al. 2000). Therefore the only possibility to observe two regions of moving outward gas projected onto the stellar limb is shown in Fig. 5. The proposed large inclination angle $i_{\mathrm{m}}$ of the stellar magnetic axis is in agreement with Muzerolle et al. (2001) who found $i_{\mathrm{m}} \simeq 70^{\circ}$ modelling $\mathrm{H}$ I Balmer and Na I D line profiles. We suggest that the LV absorption component originates in the gas, which initially moves outwards from the accretion disk along magnetic field lines and later falls onto the stellar surface. In other words the LV region is the part of the accretion column that projects onto the stellar limb. We did not observe redshifted Fe II lines nor Mg II redshifted absorption features because at large distance from the stellar surface the inflowing matter projects out of the stellar limb. At the moment of the observation and at distance $<0.1 R_{*}$ these ions disappear due to photoionization (Lamzin 1998). The HV region is a real outflow, i.e. the base of the magnetospheric wind. In this picture (Fig. 5) blueshifted HV and LV Mg II absorption features should appear and disappear periodically, unless the stellar rotation axis is also strongly inclined to the disk rotational axis. A similar behavior can be probably seen in the resonant lines of $\mathrm{Na}$ I and $\mathrm{Ca}$ II as well.

\subsection{Detection of a flare and $\mathrm{Mg} / /$ line profile variability}

AB00 found that the BP Tau Mg II line flux varies in the range $0.8-8.210^{-12} \mathrm{erg} \mathrm{s}^{-1} \mathrm{~cm}^{-2}$ - see also Simon et al. (1990) and Gomez de Castro \& Franqueira (1997). We found a flux $\simeq 1.8 \times 10^{-12} \mathrm{erg} \mathrm{s}^{-1} \mathrm{~cm}^{-2}$, so the HST spectrum represents a low level of stellar activity. To investigate the reason of the doublet flux variability we plot in Fig. 6 all IUE high resolution spectra in the vicinity of $2800 \AA$ along with the HST spectrum from Fig. 1 for reference. Gomez de Castro \& Franqueira (1997) compared the same IUE spectra with the HST/GHRS one - see their Fig. 4 - but they did not recalculate vacuum wavelengths of the HST spectrum to air wavelengths. For this reason the IS absorption features in Mg II $u v 1$ lines appear redshifted in their plot and were misinterpreted as a signature of a "warm infalling gas".

Unfortunately the quality of IUE spectra is not good enough to definitely detect either HV or LV absorption features if they have the same strength as in the HST spectrum. Nevertheless it is possible to say something about the variability of $\mathrm{Mg}$ II line profiles. In LWR11130 and LWP09282 spectra the intensity of the lines was 1.5-2 times as big as one of the HST spectrum, the red wings of $\mathrm{h}$ and $\mathrm{k}$ lines increased more significantly than the blue ones. In the LWP06963 spectrum the situation is opposite: the blue part of the line profiles increased much stronger than the red ones.

According to AB00 the timescale of the Mg II $2800 \AA$ doublet flux and equivalent width variability is much less than the stellar rotation period, which means that the variability is the result of some non-stationary process rather than rotational modulation or occultation by dust clouds. The intensity of the $\mathrm{h}$ and $\mathrm{k}$ lines in the LWP09417 spectrum is close to that of the HST spectrum but the extent of their blue wings is significantly less (Fig. 6). By the way, the doublet line blue wings also look less extended in other IUE spectra in comparison with the HST spectrum. It means that the observed geometry of the gas flow is also time variable in agreement with the predictions of 


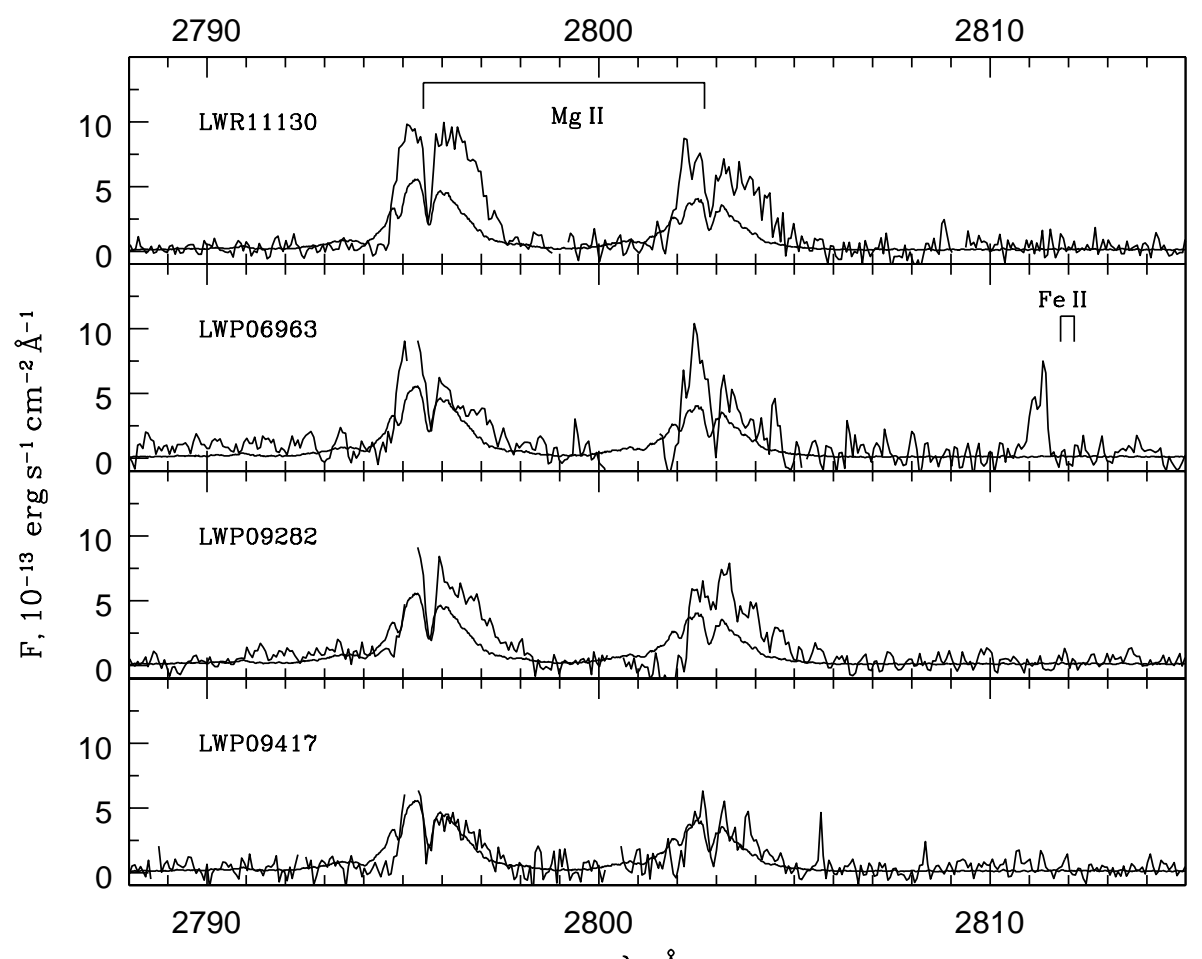

$\lambda, \AA$

Fig. 6. High resolution IUE spectra of BP Tau in the vicinity of Mg II 2800 doublet (solid lines). Gaps in the curves respect to pixels with non-zero quality flag. HST/GHRS spectrum is plotted for comparison (thin lines). Rest frame positions of the Fe II 2811.8, 2812.1 Å lines are shown - see text for details.

the inclined magnetic rotator model. Furthermore if the inclination of the stellar magnetic axis is large we can expect strong variability due to the non-stationary character of the accretion process.

In the LWP06963 spectrum a strong emission feature is present at $\lambda \simeq 2811.2 \AA$ but it is absent in all other spectra (Fig. 6). There is no reason to doubt the reality of this feature because its pixel fluxes are $4-5$ times larger than the flux errors. We identified this feature with a blend of two Fe II lines of the $c^{4} \mathrm{D}-\mathrm{s}^{4} \mathrm{D}^{\circ}$ multiplet with a common upper level $J=5 / 2(2811.81,2812.14 \AA)$ blueshifted to $\simeq 80 \mathrm{~km} \mathrm{~s}^{-1}$ relative to the rest frame position. The upper level of these lines can be pumped from the $a^{4} \mathrm{P}_{3 / 2}$ level $\left(E_{i} \simeq 1 \mathrm{eV}\right)$ by quanta from the blue wing $\left(\Delta V \simeq-230 \mathrm{~km} \mathrm{~s}^{-1}\right)$ of the $\mathrm{H} \mathrm{I} \mathrm{L} \alpha$ line. Fluorescent Fe II lines pumped by L $\alpha$ line quanta were observed in the RW Aur spectrum as well (Errico et al. 2000).

The excitation energy of the Fe II $c^{4} \mathrm{D}$ term is $E_{i} \simeq$ $7.5 \mathrm{eV}$ so the fluorescent lines are definitely optically thin and their flux ratio should be equal to the ratio of transition probabilities. According to Nahar (1995) the expected $2811.81 \AA$ line is $\simeq 2.5$ times weaker than the $2812.14 \AA$ line, in agreement with the observations. We also searched for other Fe II lines with an $\mathrm{s}^{4} \mathrm{D}_{5 / 2}^{\mathrm{o}}$ upper level which fall in the range of LWP06963 spectrum. Unfortunately they are 2-10 times weaker than the $2811.2 \AA$ emission feature and fall in regions with low $\mathrm{S} / \mathrm{N}$-ratio, so we could not identify them.

We believe that the observed increase of $\mathrm{h}$ and $\mathrm{k}$ line blue wing intensity is accompanied by the increase of hydrogen $\mathrm{L} \alpha$ line intensity, resulting in the appearance of blueshifted Fe II fluorescent lines. The BP Tau visual brightness was also large; during this flare - see Table 2 only two stellar spectra out of 49 from the INES database were observed with larger values of FES counts. In the frame of the inclined rotator model these facts can be interpreted as follows.

The increase of the accretion rate results in an increase of the continuum as well as the $\mathrm{Mg}$ II 2800 and $\mathrm{L} \alpha$ line emission from the accretion shock. We assume that the flare has occurred when the stellar magnetic pole was on the visible hemisphere of the star, with a rotational period phase $\phi$ shifted to $\Delta \phi \sim \pi$ relative to the phase shown in Fig. 5. The regions of the accretion column where gas moves away from the observer are projected onto the star limb, while the regions with negative radial velocities project out of the limb. Thus the red wings of $\mathrm{h}$ and $\mathrm{k}$ lines are attenuated due to the absorption inside the accretion column and the intensity of the blue wings is increased. In other words we also observe an "absorption feature" in $\mathrm{h}$ and $\mathrm{k}$ lines, but it is redshifted and much wider than the LV feature because, apparently, the radial velocity range is larger.

It was shown in Sect. 3 that the accretion shock $\mathrm{L} \alpha$ line has a deep central depression, i.e. its profile is double peaked. During the flare only those regions of the external gas flow could produce observed Fe II fluorescent lines which had a Doppler shift of $-230 \mathrm{~km} \mathrm{~s}^{-1}$ relative to one of the two peaks of the accretion shock $\mathrm{L} \alpha$ line. If we suppose it was the red peak, then the Fe II fluorescent line 
formation region was located in the stellar wind or in that part of the accretion column where gas moved away from the star (Fig. 5). This is the reason of the blueshift observed in the iron lines.

The energy release was very large during the flare. The observed flux of the Fe II 2811.2 emission feature is $\simeq 3 \times 10^{-13} \mathrm{erg} \mathrm{s}^{-1} \mathrm{~cm}^{-2}$ and its dereddened luminosity is $\simeq 2 \times 10^{30}$ erg s $\mathrm{s}^{-1}$, assuming $D=140 \mathrm{pc}$, $A_{V} \simeq 0.5^{\mathrm{m}}$ and the normal extinction law (Seaton 1979). Meanwhile the lines of the $b^{4} \mathrm{D}-\mathrm{s}^{4} \mathrm{D}^{0}$ multiplet (1547.24, 1547.80 and $1550.09 \AA$ ) have a transition probability $\sim 30$ times larger than that of the $c^{4} \mathrm{D}-\mathrm{s}^{4} \mathrm{D}^{0} \mathrm{mul}-$ tiplet which is the largest value among Fe II lines with an $\mathrm{s}^{4} \mathrm{D}_{5 / 2}^{0}$ upper level. The excitation energy of the $\mathrm{b}^{4} \mathrm{D}$ term is high enough $\left(E_{i} \simeq 3.9 \mathrm{eV}\right)$ to expect that the lines of this multiplet are optically thin. Thus the luminosity of all iron fluorescent lines originating from the $\mathrm{s}^{4} \mathrm{D}_{5 / 2}^{0}$ upper level was near $3 \%$ of the BP Tau total luminosity $L_{*} \simeq 0.9 L_{\odot}$ (Gullbring et al. 1998). Obviously only a small fraction of $\mathrm{L} \alpha$ quanta was re-radiated in the Fe II fluorescent lines, so the total excess L $\alpha$ luminosity at the moment of the LWP6963 observation was comparable with $L_{*}$, and $L_{\text {ac }}$ apparently even exceeded $L_{*}$. In the case of RU Lupi Lamzin et al. (1996) also observed a flare like event with an excess luminosity larger than the stellar bolometric luminosity.

A large energy release in Fe II fluorescent lines means that the optical depth $\tau_{0}$ of the iron $1214.73 \AA$ pumping line $\left(\mathrm{a}^{4} \mathrm{P}_{3 / 2}-\mathrm{s}^{4} \mathrm{D}_{5 / 2}^{0}\right.$ transition $)$ exceeds unity - we take $\tau_{0}=3$ as a lower limit. Let $n_{i}$ be the relative population of the $\mathrm{a}^{4} \mathrm{P}_{3 / 2}$ level, $n_{\mathrm{Fe} \text { II }}$ the relative abundance of the $\mathrm{Fe}^{+}$ ion, $N_{\mathrm{H}}$ the hydrogen (both ionized \& neutral) particle density $\left(\mathrm{cm}^{-3}\right)$ and $l$ the radial extent of the line formation region. We have

$\tau_{0}=k_{0} n_{i} n_{\mathrm{Fe} \text { II }} \xi_{\mathrm{Fe}} N_{\mathrm{H}} l \geq 3$

where $k_{0}$ is the absorption cross section in the center of the line and $\xi_{\mathrm{Fe}} \simeq 4 \times 10^{-5}$ is the iron relative abundance (Allen 1973). Assuming that the local line broadening is due to thermal motion and adopting the atomic data by Nahar (1995) we can derive from Eq. (9.43) of Mihalas (1978) that $k_{0} \simeq 4.5 \times 10^{-16} / \sqrt{T / 10^{4}} \mathrm{~cm}^{2}$, where $T$ is the gas temperature. As long as $n_{\mathrm{Fe} \text { II }} \leq 1$ we find that, during the flare, the hydrogen column density $N_{\mathrm{H}} l$ is larger than $1.5 \times 10^{20} / n_{i} \mathrm{~cm}^{-2}$. Adopting statistical weights and excitation energies of the Fe II levels by the NIST Database (http://physics.nist.gov) we find that with $T<10^{4} \mathrm{~K}$ Boltzman (LTE) population of the $\mathrm{a}^{4} \mathrm{P}_{3 / 2}$ level is less than 0.014 , it is the upper limit to the $n_{i}$-value. Therefore during the flare $N_{\mathrm{H}} l$ was $\geq 10^{22} \mathrm{~cm}^{-2}$.

From the Cruddace et al. (1974) data we find that at this moment the BP Tau X-ray emission should be strongly attenuated below $1 \mathrm{keV}\left(\tau_{1 \mathrm{kev}} \gtrsim 2\right)$ if the iron fluorescent line formation region is projected onto the stellar limb. There is direct evidence that in some cases CTTS $\mathrm{X}$-ray variability can be due to circumstellar envelope variable extinction (Walter \& Kuhi 1981; Kastner et al. 1999).
The soft X-ray emission of the CTTS accretion shock is practically independent on the infall gas density (Lamzin 1999), i.e. the theory predicts the absence of a correlation between the variations in optical and X-ray bands in agreement with the observations of BP Tau (Gullbring et al. 1997).

\section{Conclusions}

The main results of our investigation can be summarized as follows.

- BP Tau activity can be explained in the frame of a disk accretion model if we assume that the stellar magnetic axis is strongly inclined to the disk plane. In the frame of this hypothesis, the nontrivial character of line flux and profile variability is the result of the nonstationary accretion observed at different orientations of the stellar magnetosphere relative to the Earth.

- We found the following average parameters of BP Tau accretion process: relative surface area of the accretion zone $f \simeq 0.25$, accretion rate $\dot{M}_{\mathrm{ac}} \simeq 3.6 \times$ $10^{-8} M_{\odot} \mathrm{yr}^{-1}$, accretion energy flux $\mathcal{F}=2.3 \times$ $10^{10} \mathrm{erg} \mathrm{s}^{-1} \mathrm{~cm}^{-2}$ and accretion luminosity $L_{\mathrm{ac}} \simeq$ $0.37 L_{\odot}$. All values were derived assuming that the accretion is homogeneous, i.e. the infall gas density and infall gas velocity are the same in all points of the accretion zone.

- Ardila \& Basri (2000) found that the accretion spot surface area is indeed proportional to the square of the accretion rate, applying the CG98 accretion shock model. We argue that this model is too crude to believe their result.

- A strong flare in the Fe II 2811.8, 2812.1 $\AA$ lines was detected. It was probably produced by an increase of the accretion rate. During the flare, the accretion luminosity was comparable to or even larger than the stellar bolometric luminosity.

- Finally we identified a number of lines in the UV spectra of BP Tau with $\mathrm{H}_{2}$ lines.

We were strongly restricted by the quantity and quality of analysed data. It is necessary to observe the star in a wider UV spectral range and at different phases of the rotational period in order to get information on the geometry and parameters of the BP Tau accretion shock. Fe II fluorescent lines, pumped by accretion shock $\mathrm{L} \alpha$ radiation, can be used as a tracer of the gas flow in the vicinity of the star.

Acknowledgements. This work was accomplished during the stage of S. L. at Astronomical Observatory of Capodimonte (OAC) in Naples. S. L. thanks the OAC staff for support as well as the Russian Fund of Fundamental Research for the grant 99-02-17184. The authors thank the anonymous referee for his helpful remarks.

\section{References}

Abgrall, H., Roueff, E., Launay, F., Roncin, J.-Y., \& Subtil, J. L. 1993, A\&AS, 101, 273 
Allen, C. W. 1973, Astrophysical Quantities (Athlone Press) Alencar, S. H. P., \& Basri, G. 2000, AJ, 119, 1881

Ardila, D. R., \& Basri, G. 2000, ApJ, 539, 834

Bernacca, P. L., Lattanzi, M. G., Porro, I., Neuhauser, R., \& Bucciarelli, B. 1995, A\&A, 299, 933

Cabrit, S., Edwards, S., Strom, S. E., \& Strom K. M. 1990, ApJ, 354, 687

Calvet, N., \& Gullbring, E. 1998, ApJ, 509, 802

Cruddace, R., Paresce, F., Bowyer, S., \& Lampton, M. 1974, ApJ, 187, 497

Edwards, S., Hartigan, P., Ghandour, L., \& Andrulis C. 1994, AJ, 108, 1056

Errico, L., Lamzin, S. A., \& Vittone, A. A. 2000, A\&A, 357, 951

ESA, 1997, The Hipparcos and Tycho Catalogues, ESA SP1200

Fernandez, M., Ortiz, E., Eiroa, C., \& Miranda, L. F. 1995, A\&AS, 114, 439

Gomez de Castro, A. I., \& Franqueira, M. 1997, ApJ, 482, 465

Gomez de Castro, A. I., \& Lamzin, S. A. 1999, MNRAS, 304, L41

Gullbring, E., Petrov, P. P., Ilyin, I., et al. 1996, A\&A, 314, 835

Gullbring, E., Barwig, H., \& Schmitt, J. H. M. M. 1997, A\&A, 324, 155

Gullbring, E., Hartmann, L., Briceno, C., \& Calvet, N. 1998, ApJ, 492, 323

Hartigan, P., Edwards, S., \& Ghandour, L. 1995, ApJ, 452, 736

Hartmann, L., Stahler, S., \& Mathieu, R. D. 1986, ApJ, 309, 275

Herbig, G. H., \& Bell, K. R. 1988, Lick Observ. Bull., 1111

Johns, C. M., \& Basri, G. 1995, AJ, 109, 2800

Johns-Krull, C. M., Valenti, J. A., Hatzes, A. P., \& Kanaan, A. 1999a, ApJ, 510, L41
Johns-Krull, C. M., Valenti, J. A., \& Koresko, C. 1999b, ApJ, 516,900

Johns-Krull, C. M., Valenti, J. A., \& Linsky, J. L. 2000, ApJ, 539,815

Kastner, J. H., Huenemoerder, D. P., Schultz, N. S., \& Weintraub, D. A. 1999, ApJ, 525, 837

Lamzin, S. A., Bisnovatyi-Kogan, G. S., Errico, L., et al. 1996, A\&A, 306, 877

Lamzin, S. A. 1998, Astron. Rep., 42, 322

Lamzin, S. A. 1999, Astron. Lett., 25, 430

Lamzin, S. A. 2000a, Astron. Lett., 26, 225

Lamzin, S. A. 2000b, Astron. Lett., 26, 589

Lamzin, S. A. 2000c, Astron. Rep., 44, 323

Lamzin, S. A., Vittone, A. A., \& Errico, L. 2001, Astron. Lett., 27,313

Mihalas, D. 1978, Stellar Atmospheres (W. H. Freeman \& Co., San Francisco)

Mundt, R., \& Giampapa, M. S. 1982, ApJ, 256, 156

Muzerolle, J., Hartmann, L., \& Calvet, N. 1998, AJ, 116, 455

Muzerolle, J., Calvet, N., \& Hartmann, L. 2001, ApJ, in press

Nahar, S. N. 1995, A\&A, 293, 967

Nave, G., Johansson, S., Learner, R. C. M., Thorne, A. P., \& Brault, J. W. 1994, ApJS, 94, 221

Roncin, J.-Y., \& Launay, F. 1994, J. Phys. Chem. Ref. Data, 4,1

Seaton, M.J. 1979, MNRAS, 187, 73

Simon, T., Vrba, F. J., \& Herbst, W. 1990, AJ, 100, 1957

Simon, M., Dutrey, A., \& Guilloteau, S. 2000, ApJ, 545, 1034

Van Hoof, P. 1999, Atomic Line List, v 2.01, http://www .pa.uky .edu/peter/atomic

Valenti, J. A., Johns-Krull, C. M., \& Linsky, J. L. 2000, ApJS, 129,399

Walter, F. M., \& Kuhi, L. V. 1981, ApJ, 250, 254 